\title{
Material Point Method Investigations of Trauma to Fluids and Elastic Solids Due to Finite Barriers
}

\author{
J.L. Dean \\ Department of Aeronautical Engineering \\ lowa State University \\ Ames, lowa 50014 USA \\ M.W. Roth \\ Department of Physics \\ University of Northern lowa \\ Cedar Falls, lowa 50614-0150 USA \\ Paul A. Gray \\ Department of Computer Science \\ University of Northern lowa \\ Cedar Falls, lowa 50614-0507 USA
}

Received: October 4, 2008

Accepted: January 13, 2009

\begin{abstract}
A Material Point Method (MPM) algorithm is developed and utilized to investigate how the dynamics of (Langrangian) Navier-Stokes fluids as well as that of elastic solids is affected by trauma due to finite barriers. For the fluid simulations, material point particles are placed in a two dimensional pipe with various initial and boundary conditions and stationary perturbations to fluid flow. Results show that eddy currents are present not only in the wake of the perturbing object but are also responsible for disruption of laminar flow upstream from the barrier. An unfortunately relevant application for sudden finite trauma to an elastic solid involves simulations of an aircraft striking a large building under varying system conditions. The work presented here is introductory in nature; the potential ramifications and importance of continued study is discussed and emphasized.
\end{abstract}

\section{BACKGROUND AND IMPORTANCE}

Material Point Method (MPM) simulations have a wide variety of applications and can be used in computational modeling of systems having length scales spanning several orders of magnitude. Recent research on biological and geological systems has shown that fluid flow in ducts and pipes having small, abrupt obstructions can dramatically affect the behavior of the system vis a vis the character of fluid flow. In fact it is thought that the onset of certain types of heart attacks involves the transition from laminar blood flow to chaotic, which can be triggered by the presence of plaque or other sources of roughness on the vessel interior [1]. Because of the rapid change in behavior of fluid flow given even small obstructions, it seems that much could be gained by conducting computer simulations of fluidic flow around finite barriers. The MPM method conserves mass and momentum and is nicely suited to simulate a special case of the Navier-Stokes equation, which must be modified in order to deal with a 
reasonable model of fluid flow. However, the fluid-fluid viscosity interaction as well as that for the fluid-boundary present in the NavierStokes equations is absent in MPM and so we have developed an algorithm to simulate fluids which is a wedding of the two methods.

The interaction of a fluid with a finite obstruction can be thought of as a trauma to the fluid. Since a fluid has elasticity but does not support shear forces except those incurred by viscosity (for example, those at a stationary boundary for no slip conditions) it is also of interest to examine the effect of finite trauma to an elastic body. A painfully obvious application is an aircraft impacting a large building. Since September 11, 2001 many such studies have been done and we would like to augment the current body of studies by comparing simulations of aircraft - building collisions. This paper presents preliminary results for simulations of collisions which investigate structural destruction due to beam pressure wave propagation, and sets the stage for a more detailed study where we may better understand how the post - collision pressure wave propagates throughout the building. Ultimately, with sophisticated enough simulations we may be able to gain practical insight into the construction of buildings that can withstand incredibly traumatic events.

\section{COMPUTATIONAL DETAILS}

The simulation of choice for our 2D fluid flow as well as 3D collision simulations is the Material Point Method (MPM) [2] with an algorithm developed and evaluated by $Z$. Chen and R. Brannon.[2] MPM is advantageous because it doesn't require a complex algorithm to simulate collisions or material failure. Instead, MPM maps the mass and momentum of groups of particles in the simulation to a background grid and utilizes the conservation equations of mass as well as that of momentum to ultimately advance the system through time. The use of the background grid also avoids entanglement associated with other contactbased algorithms.

To begin with, the initial conditions for the simulation include a collection of particles with initial positions $\left\{\vec{x}_{p}^{0}\right\}$, velocities $\left\{\vec{v}_{p}^{0}\right\}$ and boundary conditions chosen so as to reflect the important physics of the actual system as much as possible. Next, the background grid is defined, where computational space is separated into a defined number of grid cells in which nodes are placed in each corner of each cell. The mass $M_{p}$ of each particle $p$ at time $t$ is mapped to the nodes $i$ of the grid cells based on the shape function $N_{i}\left(\vec{x}_{p}^{t}\right)$, resulting in the mass $m_{i}^{t}$ of node $i$ at time $t$ :

$$
m_{i}^{t}=\sum_{p=1}^{N p} M_{p} N_{i}\left(\overrightarrow{\mathbf{x}}_{p}^{t}\right)
$$

The same is done for the momentum $(M v)_{p}{ }^{t}$ of the particles:

$$
(m \mathbf{v})_{i}^{t}=\sum_{p=1}^{N p}(M \mathbf{v})_{p}^{t} N_{i}\left(\mathbf{x}_{p}^{t}\right)
$$

The shape functions $N_{i}\left(\vec{x}_{p}^{t}\right)$ are calculated in the following way. The coordinates of the 3D box containing a particle $p$ are normalized so that they take on extreme values of 0 or 1 at vertices of the box. So if a particular box has a vertex at $\left(x_{j}, y_{j}, z_{j}\right)$ and another vertex at $\left(x_{j}+\Delta x, y_{j}+\Delta y, z_{j}+\Delta z\right)$ across its diagonal, the normalized coordinates are

$$
\begin{aligned}
& \zeta=\frac{\left(x_{p}^{t}-x_{j}\right)}{\Delta x} \\
& \eta=\frac{\left(y_{p}^{t}-y_{j}\right)}{\Delta y} \\
& \chi=\frac{\left(z_{p}^{t}-z_{j}\right)}{\Delta z}
\end{aligned}
$$

and the eight shape functions used in the particle-to-node mappings are calculated from the normalized coordinates as shown in Table 1 so that they are equal to unity for a vertex (node) where the particle is at and is zero for a given node if the particle is at any other node.

Details for the 2D setup are easily obtainable from the 3D information above and are given elsewhere [2]. Once the mappings in equations (1) and (2) are completed then the forces at each node must be calculated. The internal force $\left(f_{i}^{t}\right)$ 


\begin{tabular}{|l|l|}
\hline $\begin{array}{l}\text { Node } \\
\text { Coordinates }\end{array}$ & Shape Function \\
\hline$(0,0,0)$ & $(1-\xi)(1-\eta)(1-\chi)$ \\
\hline$(1,0,0)$ & $\xi(1-\eta)(1-\chi)$ \\
\hline$(1,1,0)$ & $\xi \eta(1-\chi)$ \\
\hline$(0,1,0)$ & $(1-\xi) \eta(1-\chi)$ \\
\hline$(0,0,1)$ & $(1-\xi)(1-\eta) \chi$ \\
\hline$(1,0,1)$ & $\xi(1-\eta) \chi$ \\
\hline$(1,1,1)$ & $\xi \eta \chi$ \\
\hline$(0,1,1)$ & $(1-\xi) \eta \chi$ \\
\hline
\end{tabular}

Table 1. Expressions for the eight shape functions used to map particle properties to each of the eight vertices of the grid cube it resides in.

arises from elastic deformation of the object and is calculated as

$$
\left(\vec{f}_{i}^{t}\right)^{\mathrm{int}}=-\sum_{p=1}^{N p} \mathbf{G}_{i}\left(\mathbf{x}_{p}^{t}\right) \bullet \mathbf{s}_{p}^{t} \frac{M_{p}}{\rho_{p}^{t}}
$$

The external force is then calculated at each node

$$
\vec{f}_{i}^{e x t}=\vec{c}_{i}^{t}+M_{p} \vec{g} m_{i}^{t}
$$

and then the nodal accelerations are obtained using $a_{i}^{t}=\frac{f_{i}^{t}}{m_{i}^{t}} \quad$ where the total force is given by $f_{i}^{t}=\left(f_{i}^{t}\right)^{\mathrm{int}}+\left(f_{i}^{t}\right)^{e x t}$. The node momenta are then updated in time

$$
(m v)_{i}^{t+\Delta t}=(m v)_{i}^{t}+f_{i}^{t} \Delta t
$$

and subsequently the kinematic variables associated with each particle $p$ in the simulation are updated using node - to - particle mappings:

$$
\begin{gathered}
\vec{a}_{p}^{t}=\sum_{i=1}^{N n} \frac{\vec{f}_{i}^{t}}{m_{i}^{t}} N_{i}\left(\vec{x}_{p}^{t}\right) \\
\vec{v}_{p}^{t}=\sum_{i=1}^{N n} \frac{(m \vec{v})_{i}^{t+\Delta t}}{m_{i}^{t}} N_{i}\left(\vec{x}_{p}^{t}\right) \\
\vec{x}_{p}^{t+\Delta t}=\vec{x}_{p}^{t}+\vec{v}_{p}^{t+\Delta t} \Delta t
\end{gathered}
$$

The new particle kinematic variables in equations (7)-(9) are mapped back onto the nodes to obtain updated nodal velocities.

Now because the simulated material(s) may have deformed in being updated, the elastic properties of the system must be taken into account. The rate of strain tensor $\vec{e}_{\text {is }}$ now calculated from the velocity gradients: $e_{i j}=\left(\partial_{j} x_{j}^{\prime}+\partial_{i} x_{j}^{\prime}\right)$. Here the primed variables represent velocity components and, explicitly in 3D we have

$\vec{e}=\left[\begin{array}{ccc}\frac{\partial u}{\partial x} & \frac{1}{2}\left(\frac{\partial u}{\partial y}+\frac{\partial v}{\partial x}\right) & \frac{1}{2}\left(\frac{\partial u}{\partial z}+\frac{\partial w}{\partial x}\right) \\ \frac{1}{2}\left(\frac{\partial v}{\partial y}+\frac{\partial v}{\partial x}\right) & \frac{\partial v}{\partial y} & \frac{1}{2}\left(\frac{\partial v}{\partial z}+\frac{\partial w}{\partial y}\right) \\ \frac{1}{2}\left(\frac{\partial u}{\partial z}+\frac{\partial w}{\partial x}\right) & \frac{1}{2}\left(\frac{\partial u}{\partial y}+\frac{\partial u}{\partial y}\right) & \frac{\partial w}{\partial z}\end{array}\right]$

Subsequently, the $3 d$ elastic stress-strain relationship is utilized to calculate the updated stress tensor $\vec{s}^{t+\Delta t}=\vec{s}^{t}+\Delta \vec{s}$, where $\Delta s_{i j}=\sigma_{i j}=\sum_{i j k l} C_{i j k l} \varepsilon_{k l}$. Because the strain tensor in eqn. (10) is symmetric, it in general yields six nonsuperfluous elements and the stiffness tensor $C_{i j k l}$ as well as the displacement tensor $\varepsilon_{k l}$ are cast in a $6 \times 6$ format: 


$$
\left[\begin{array}{l}
\sigma_{x} \\
\sigma_{y} \\
\sigma_{z} \\
\tau_{x y} \\
\tau_{y z} \\
\tau_{z x}
\end{array}\right]=\frac{E}{(1+v)(1-2 v)}\left[\begin{array}{cccccc}
1-v & v & v & 0 & 0 & 0 \\
v & 1-v & v & 0 & 0 & 0 \\
v & v & 1-v & 0 & 0 & 0 \\
0 & 0 & 0 & \frac{1-2 v}{2} & 0 & 0 \\
0 & 0 & 0 & 0 & \frac{1-2 v}{2} & 0 \\
0 & 0 & 0 & 0 & 0 & \frac{1-2 v}{2}
\end{array}\right]\left[\begin{array}{c}
\varepsilon_{x} \\
\varepsilon_{y} \\
\varepsilon_{z} \\
\gamma_{x y} \\
\gamma_{y z} \\
\gamma_{z x}
\end{array}\right]
$$

The algorithm then returns to mapping particle properties to the grid (eqn. 1) and is repeated until a desired time limit is reached.

To simulate a fluid, all the nondiagonal elements are removed from equation (11) and fluid-fluid (particleparticle) interaction terms are added in the simulation. The boundary layer behavior may be modeled by either adding another interaction or by enforcing particle velocity boundary conditions.

\section{RESULTS AND DISCUSSION}

\section{a. Case 1:2D Fluid Simulation}

For fluid simulations "fluid particles" are arbitrarily placed within a two dimensional pipe with a uniform initial velocity. A specific amount of particles are maintained throughout the simulations by utilizing periodic boundary conditions (resetting particles who have reached the end of the pipe). A single stationary boundary of arbitrary shape is also placed in the wake of the fluid medium as a perturbation to the fluid flow. For all test cases, fluid particles are confined within the pipe wall defined by light gray lines (Figure 1). Several simulations are necessary to check particular fluid behavior (i.e., viscous interactions among fluid elements, localized vorticity, and fluid 'mixing'). The first test investigates viscous interaction between fluid elements as well as overall fluid mixing to aid in verification of fluid-like behavior among elements. Results shown in Figure 1 are indicative of predicted viscous behavior, and over-all particle mixing is directly due to viscous shear force interactions between fluid elements. In panel (a) initial placement of particles are such that the stationary boundary will immediately cause disturbance in the fluid medium. Viscous interactions become apparent in deformations appearing within the fluid's initial condition. In panel (b) viscous force interactions become increasingly apparent in the deformation of the material. Panel (c) shows Particle grouping is apparent in front of boundary with a shape similar to that of initial placement deformation shown in (b) and fluid mixing is apparent elsewhere. In subsequent panels viscous interactions cause complete mixing of the fluid.

To properly simulate ideal fluids and investigate fluid vortices, an assumption must be made that the fluid fills the entire region being investigated. This test is an attempt to satisfy that assumption, and investigate fluid wake behind the boundary. To emphasize rotation, particles displaying clockwise rotation appear red while counterclockwise rotation is indicated with blue. Initial conditions are similar to that of the previous test, but given an initial vertical velocity to further emphasize rotation near one end of the stationary boundary. Vorticity is indeed present along the stationary boundary and in the fluid wake, but only in localized locations due to a failure to maintain the assumption that the entire region is filled with fluid. In fact, a flaw regarding MPM and fluid simulations is realized here in that it is difficult to achieve an entirely filled region with elemental particles of arbitrary size and shape.

Figure 2 shows the interaction of a fluid having two nonzero velocity components with a stationary rectangular boundary. Panel (a) shows initial placement of fluid particles throughout the entire region; rotation is immediately present for particles 


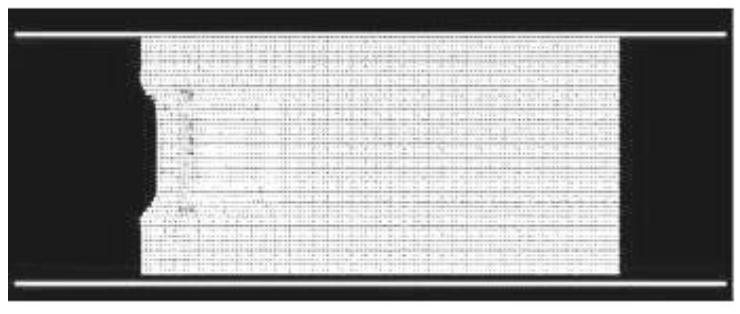

(a)

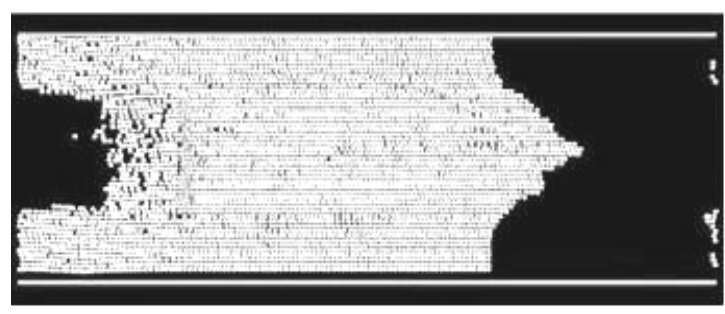

(b)

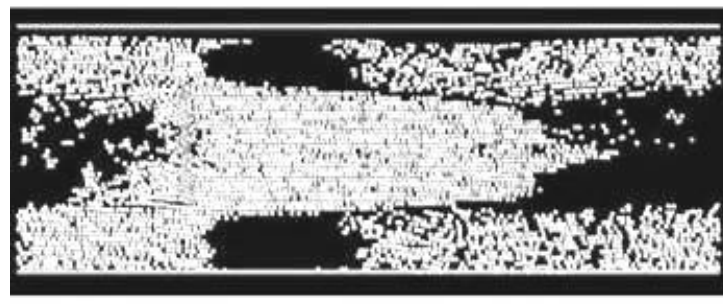

(c)

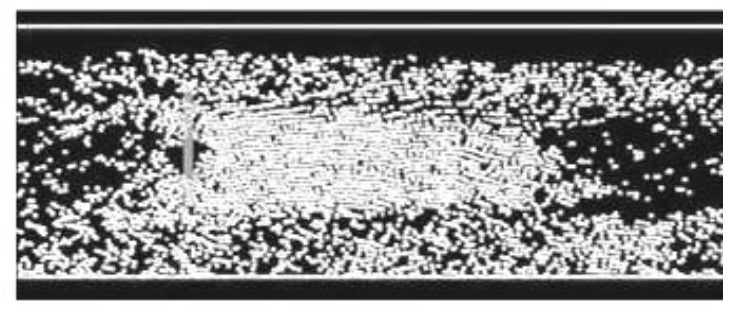

(d)

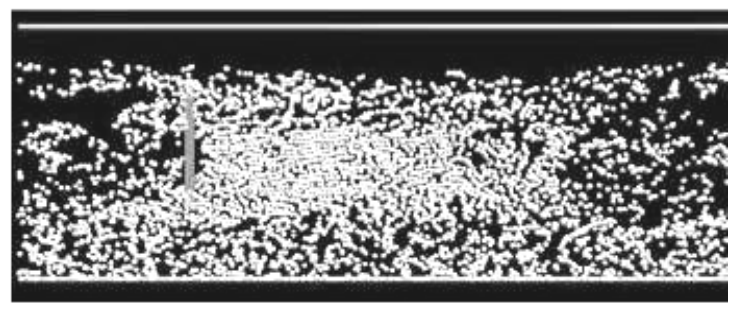

(e)

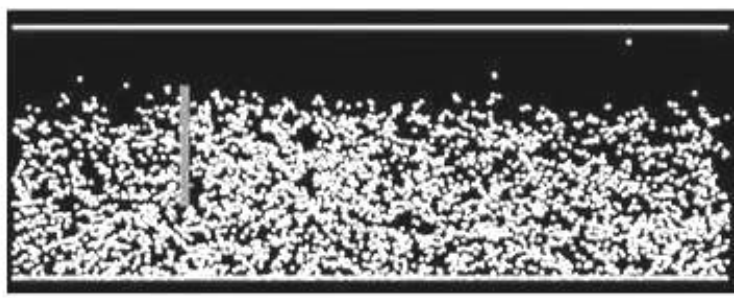

(f)

Figure 1. Simulated horizontal flow past a rectangular barrier.

that have contacted the stationary boundary. In panel (b) there is failure to maintain a completely filled region. However, rotation is also realized in localized regions in the fluid wake, particularly behind the bottom edge of the boundary. The next panel (c) shows that localized vorticity is still present among fluid particles that have interacted with the stationary boundary along the bottom surface. For further investigation on rotation and fluid vorticity and general fluid behavior, a circular boundary is swapped with the rectangular boundary, while the fluid particles initial conditions are similar to that of previous simulations with an initial uniform vertical velocity [3]. Investigation of fluid behavior beyond a circular disturbance predicts periodic groupings of localized vorticity along the wake of the fluid beyond the boundary. Panel (a) shows the initial condition at the start of the simulation, and space is not completely filled with particles due to similar issues raised in the previously discussed rectangular boundary simulations. The next panel (b) shows very slight localized rotation is present beyond the boundary, similar to that in Figure 2 . Viscous interactions in front of the boundary are also present, and influence particles before they pass or interact with the boundary. Subsequently, in panel (c) periodic groupings of localized vorticity appears to be present along the wake of the fluid.

b. Case 2: Building structural destruction due to pressure-wave propagation

The next simulation investigates structural destruction and pressure wave 


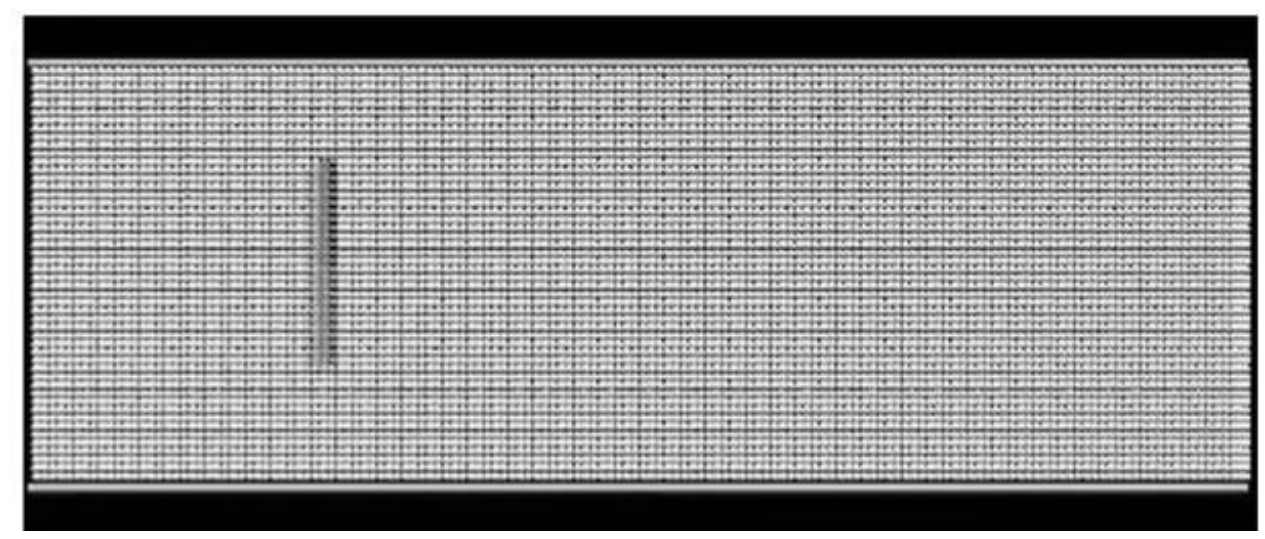

(a)

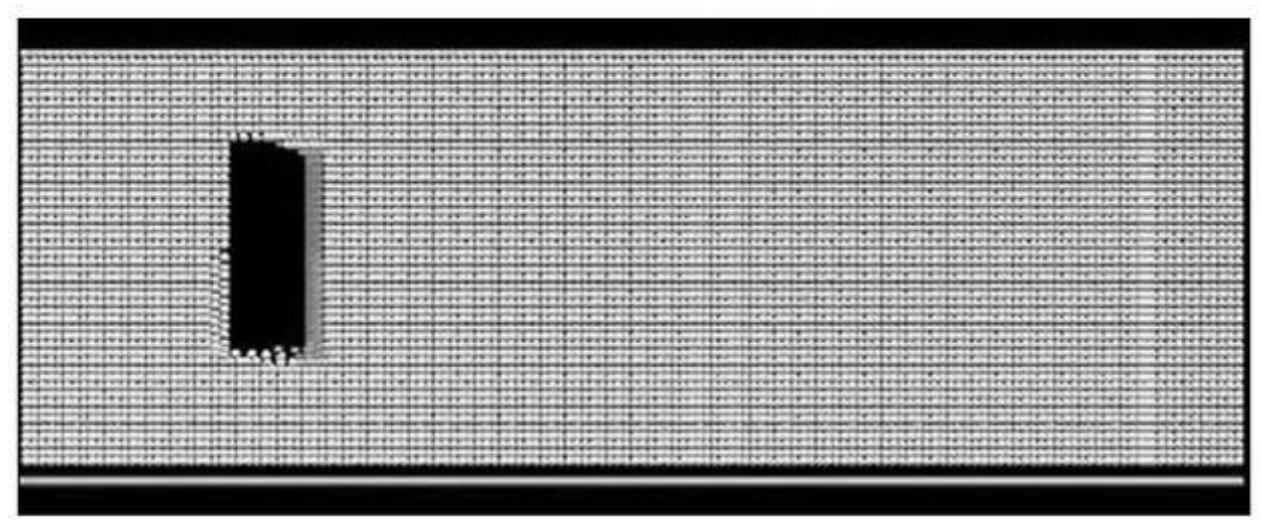

(b)

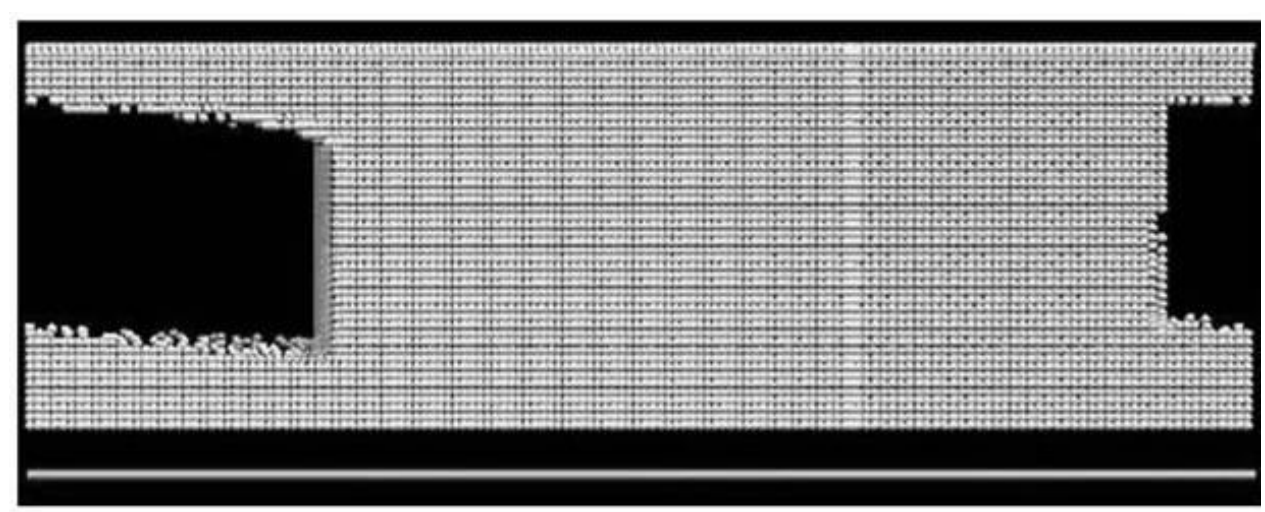

(c)

Figure2. Simulated fluid flow past a rectangular boundary with a non-zero initial vertical velocity.

propagation through a structure due to trauma from a unique shape, similar to that of the September $11^{\text {th }}$ attack. Material particles within the building are placed such that they combine to form individual vertical beams spanning the length of the structure.
Each beam contains fifty beam 'particles' stacked on top of each other with 100 beams total. Gravitational forces are not present due to issues regarding structural integrity prior to collision, and to isolate forces to those only due to collision. This 


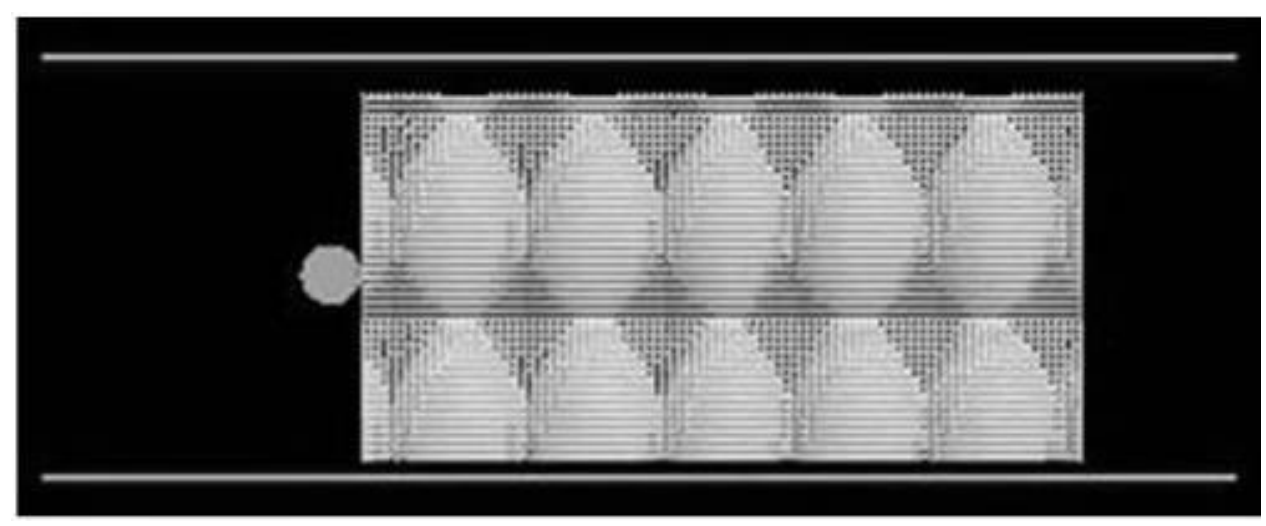

(a)

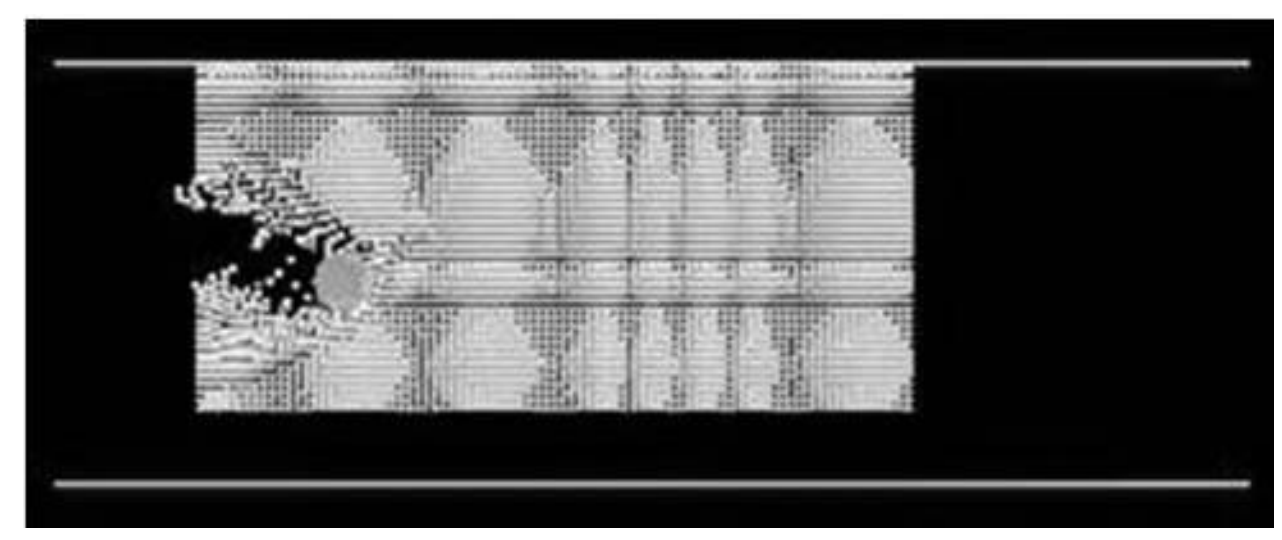

(b)

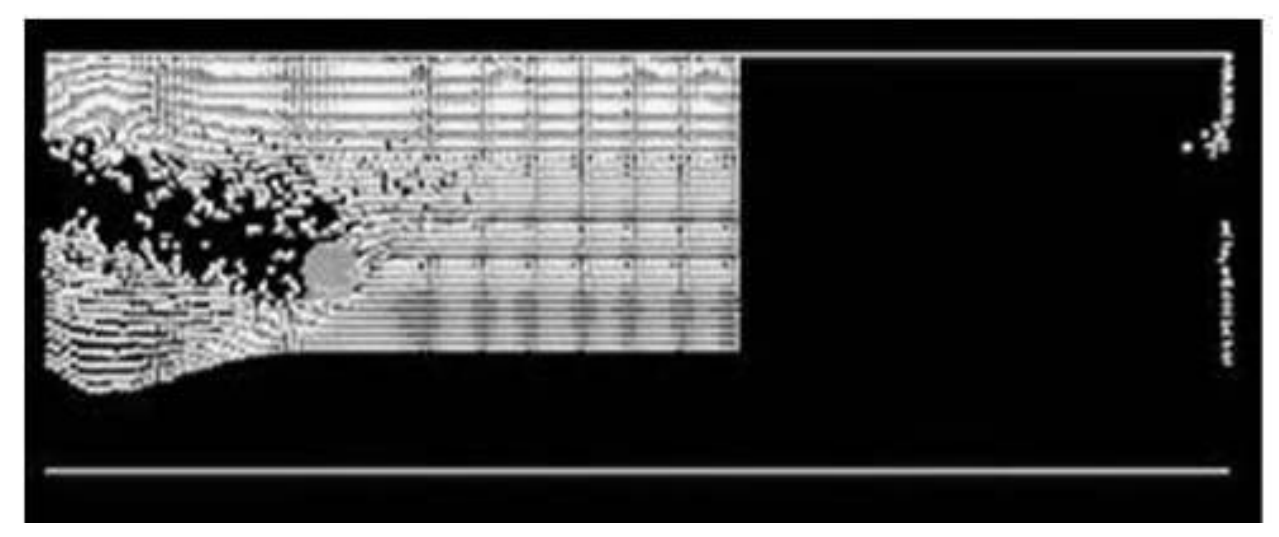

(c)

Figure 3. Simulated fluid flow past a circular boundary with initial vertical velocity.

simulation is utilized to promote investigation of structural failure due to irregular shaped body collisions. Case 2 investigates the destruction upon the building caused by the initial plane collision, as well as pressure propagation to create a general structural failure. Panel (a) shows the initial simulation set up, and panel (b) illustrates the initial impact of the aircraft into the building structure. The aircraft's fuselage generates 

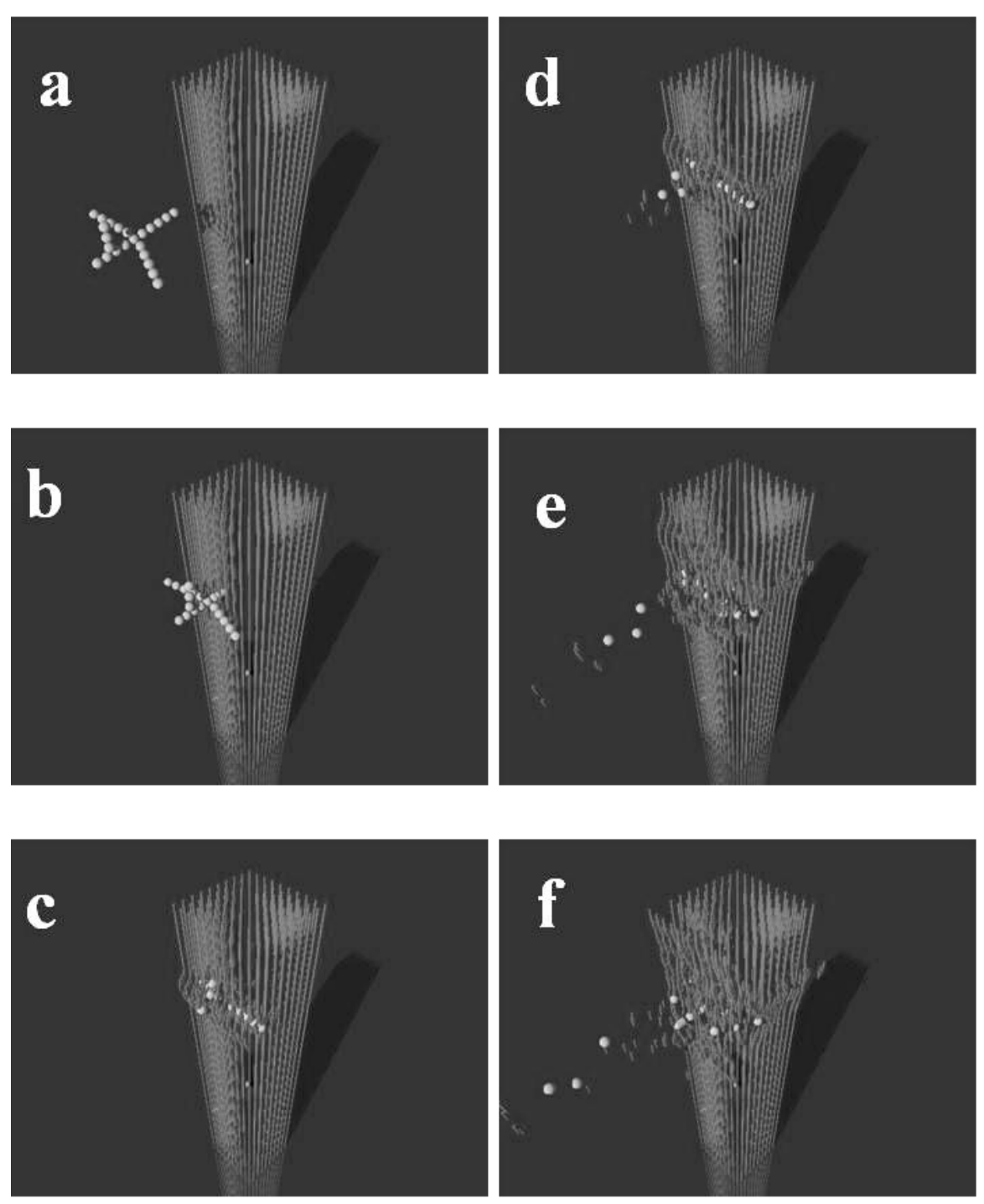

Figure 4. Simulated aircraft-building collision. Note the pressure wave propagation throughout structure and over all destruction.

relatively little impact to the structure as a whole. Panel (c) shows secondary impact due to aircraft wings and tail. Pressure wave is generated due to this collision and begins propagating up and down the surface of the structure at the point of impact. In panel (d) a longitudinal forwardly propagating pressure wave bisects the structure that propagates from the wings. This pressure wave produces the highest level of general destruction within the building. Note: some particles depart from the structure due to reverberant forces due to impact. In subsequent panels the latitude pressure wave reaches the top of the building and begins to propagate in the opposite direction. The longitudinal pressure wave begins to spread vertically causing serious structural issues throughout the base of the structure, effectively 'slicing' the building.

As shown, the fuselage generates relatively little overall destruction of the building, due to the structure being comprised of only vertically stacked beams. The wing sections of the aircraft create the highest level of destruction through a generation of pressure waves that propagate vertically and horizontally through the building. Further investigation regarding 
beam strength, aircraft integrity, and outdoor conditions is necessary to improve this simulation.

\section{REFERENCES}

1. http://www.womensheart.org/content/ HeartAttack/what_is_a_heart_attack.asp

2. Z Chen, RM Brannon, Sandia National Laboratories (SAND2002-0482), 2002

3. http://www.mame.mu.oz.au/fluids/
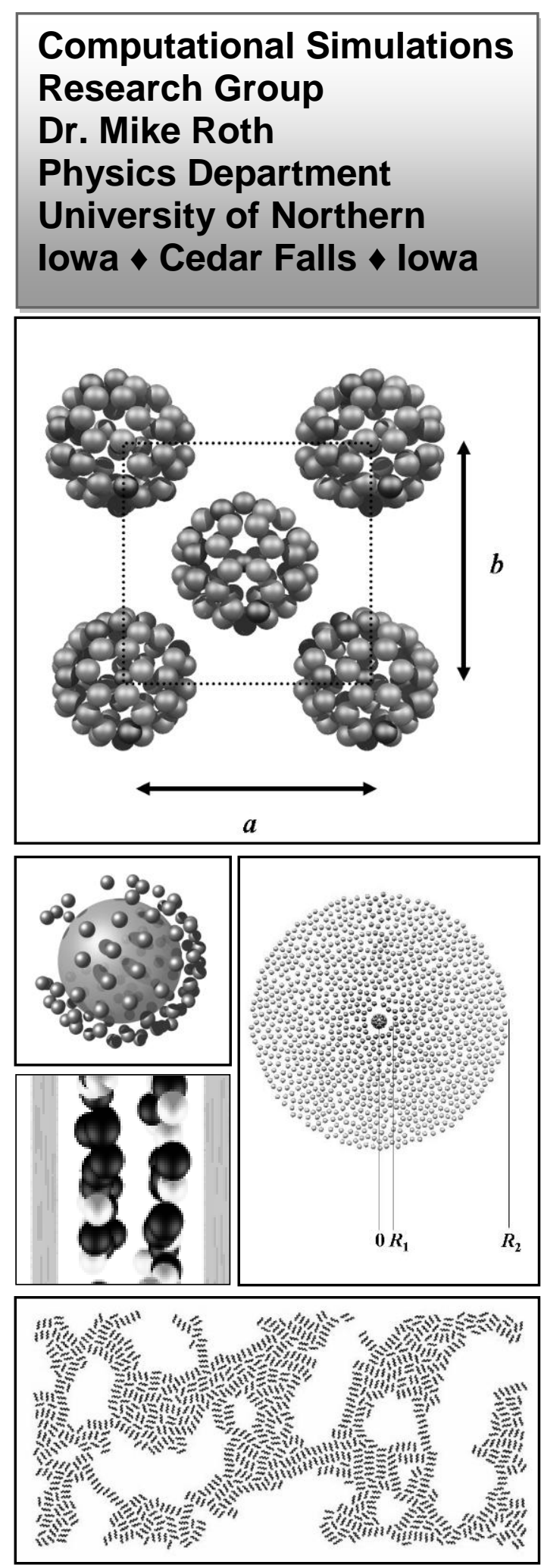

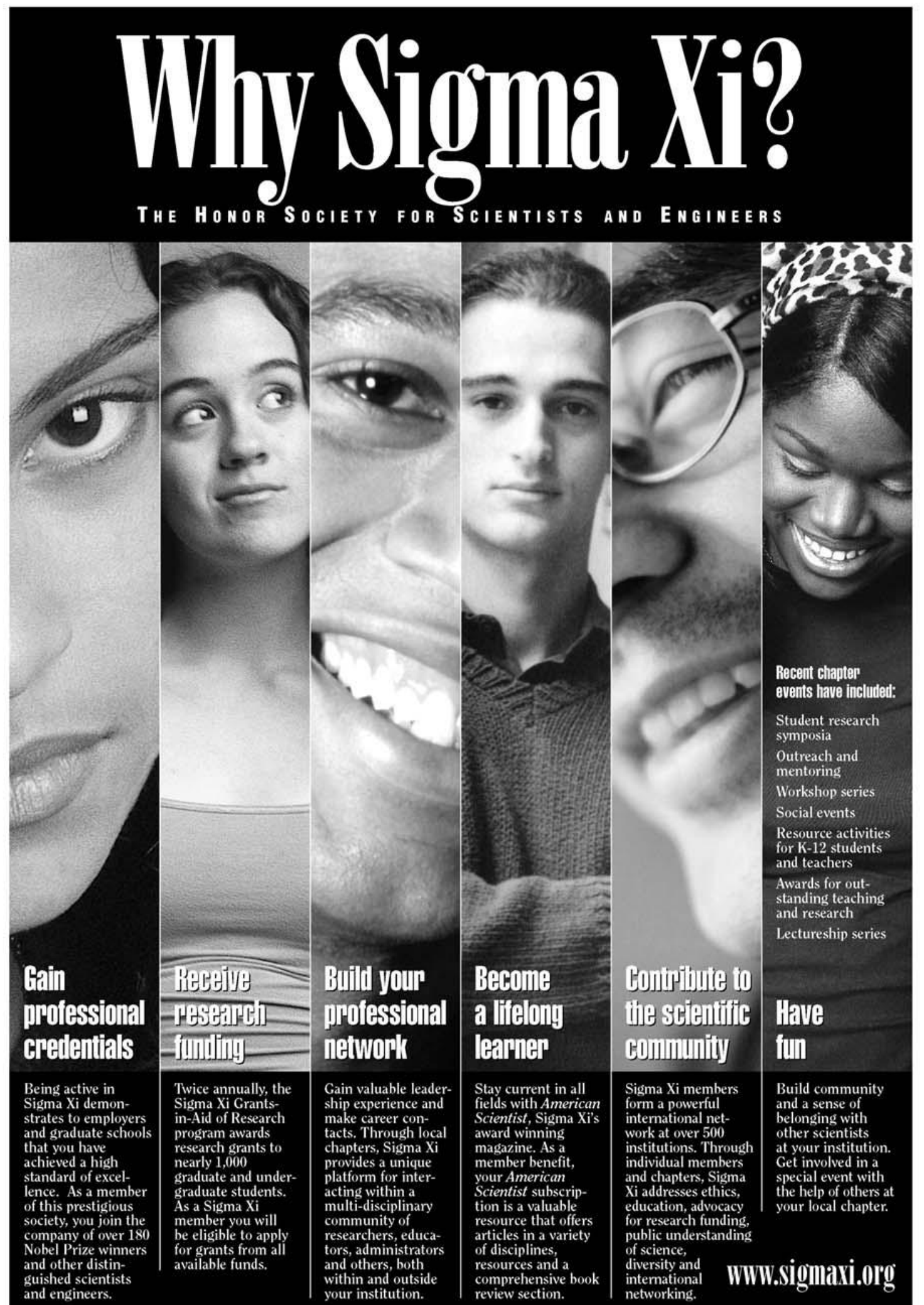

Sigma Xi, The Scientific Research Society • 88 Alexander Drive • P.O. Box 13975 • Research Triangle Park, NC 27708 • 819-549-4691 • 800-243-6534 